\title{
The surface electromyography noise filtering and unwanted recordings attenuation for lower limb robotic system
}

\author{
Abdelhakim Deboucha \\ Department of Electrical Engineering, Ecole Superieure des Science Appliquees-Alger, Algiers, Algeria \\ Innovative Manufacturing, Mechatronics and Sports Lab, University Malaysia Pahang, Pahang, Malaysia
}

\begin{tabular}{l} 
Article Info \\
\hline Article history: \\
Received Sep 26, 2021 \\
Revised Dec 25, 2021 \\
Accepted Dec 30, 2021 \\
\hline Keywords: \\
Adaptive cutoff frequency \\
Butterworth filter \\
Robotic system \\
Surface electromyography \\
Unwanted recordings
\end{tabular}

Unwanted recordings

\begin{abstract}
Exoskeleton robotic device (ERD) for rehabilitation purposes, physically interacts alongside with the user where high cognitive interaction and the safe human-machine system is required. To ensure safe interaction, there is a need to detect the user's motion intention. One of the bio-signals that have been found to reflect directly the individual's motion intention is surface electromyography (sEMG). However, sEMG signals are inevitably full of noises, not to mention the unwanted recordings and other artifacts between muscles where they cannot be freely used as a control signal for ERD. This paper presents the use of the Butterworth filter for noise suppression and the attenuation of unwanted recordings. Using classical Butterworth filter typically is unable to eliminate or attenuate the unwanted contamination on the signal of interest to its baseline level. Therefore, it is critical to modify the Butterworth filter at this stage. sEMG signals from the biceps femoris and rectus femoris muscles of seven healthy male young adults were recorded in this study. The onset/offset technique is utilized to detect the presence of the additional signal contaminated on the signal of interest. If the onset/offset index points are not approximately correlated with the movement, this means there is a contaminated measurement on the signal of interest. At this interval, a filter with distributed cutoff frequency plays the role to have the already smoothed baseline signal. In summary, the modified Butterworth filter shows to have a good performance to suppress the noises and to attenuate the unwanted recordings adaptively which ensures a safe human-machine system.
\end{abstract}

This is an open access article under the CC BY-SA license.

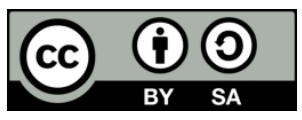

\section{Corresponding Author:}

Abdelhakim Deboucha

Department of Electrical Engineering, Ecole Superieure des Science Appliquees-Alger

Boîte Postale 474, Place des Martyrs, Algiers, Algeria

Email: a.deboucha@g.essa-alger.edu.dz

\section{INTRODUCTION}

Rehabilitation devices such as powered exoskeleton alter directly on the individual/patient to overcome some limitations [1]. Exoskeletons are intermingled assistive devices in terms that they can be assistive devices until the individual recover back his/her motor skill. In order to ensure safe physical interaction with high cognitive, there is a need to detect the user's movement intention. Several researchers such as [2]-[4], have significantly deepened our understanding and open interest in the use of surface electromyography to control assistive devices. Indeed, sEMG amplitude is often used to trigger the motion of the robotic system [3], [5].

When using surface electrodes, adjacent /unrelated recordings other than the muscle of interest may also interfere with the signal. This signal contamination is often referred to as crosstalk/unwanted recordings 
which are mainly due to the pickup area of the surface electrodes. Hence, logging this signal without careful processing to the assistive device will accidentally trigger the latter and violate the cognitive interaction requirement. To result in a free contaminated signal from other artifacts, careful efforts had been undertaken into consideration such as selecting the appropriate electrode size, inter-electrode distance, and location of recordings over the muscle [6]-[8].

Despite all of these efforts, in practice, it is often difficult to selectively record from a single muscle. Unwanted recordings in sEMG have been investigated by several researchers for the purpose of clinical neuromuscular disease investigation and ergonomics diagnosis [9], [10]. In particular, intensive research on the forearm to quantify the unwanted recordings between the muscles is carried out because many muscles are tightly gathered [11], [12]. On the other hand, lower limb muscles have been investigated by a few researchers to examine the aforementioned issue. There are two methods to reduce/detect unwanted recordings contamination, firstly is when the measurement is already recorded (offline data), and secondly, is before any measurement has been done. From first sight, the most common technique used to determine the presence of unwanted recordings is known as blind source separation (BSS). This technique is essentially based on principal component analysis (PCA) and independent component analysis (ICA) as reported by [13]. ICA technique works on the assumption to have the number of sources to be separated as the number of recordings, and it is the more successful technique when these sources are enclosing different frequencies such as an electrocardiogram (ECG) and electro-oculogram (EOG). However, it is difficult to use ICA when trying to separate the neighboring muscles' activities from the signal of interest due to similar electrical properties between muscles. Cross-correlation is also a widely used tool to identify the common component between two signals [11]. Nevertheless, this latter is only useful when the two signals are known.

On the other hand, onset detection is a useful technique in sEMG analysis to detect when the EMG burst and when it is at rest [14]. Moreover, the onset technique is widely implemented to trigger robotic devices [15]. However, this technique can also be used to detect the existence of unwanted recordings when the experimental measurements are supported by the movement recordings, simultaneously. Due to less ripple in the pass-band of Butterworth filters and rapid response in the time domain, they are often recommended in the biomechanics field for the purpose of data smoothing [16]. Furthermore, Butterworth filters have gained a wide acceptance in filtering sEMG by several researchers [17], [18]. The present paper introduces the use of the movement recordings together with sEMG recordings to alter the behavior of the Butterworth filter in order to detect the onset of the sEMG. The Butterworth filter is fairly modified to be an adaptive filter only when it seems there is no movement by the individual. Recursive least squares (RLS) algorithm is utilized to modify the Butterworth filter for the purpose of attenuating the unwanted recordings which in turn ensure safe human-machine interaction.

\section{RESEARCH METHOD}

\subsection{Subject}

Seven healthy young adult subjects in total with ages ranging from 20 to 24 years old volunteered in this study. Before the experiment, each subject was informed about the nature and purpose of the study and gave written informed consent. The mean height of the subjects was $1.70 \pm 0.7$ and weight $72 \pm 4 \mathrm{~kg}$. Electromyography data were recorded from a pair of surface bipolar electrodes $(3 \mathrm{~cm}$ inter-electrode distance, metallic part: $\mathrm{Ag}-\mathrm{AgCl}$ manufacturer: Shenzhen Amydi-med Electronics Tech Co., Ltd) placed over the relevant muscles connected to an EMG sensor (size: $53 \mathrm{~mm}$ x $32 \mathrm{~mm}$ x $23 \mathrm{~mm}$, frequency range: 5 to $482 \mathrm{~Hz}$, max signal range: $4.4 \mathrm{mV}$, manufacturer: Shimmer discovery in motion). Kinematics data were obtained using a Gyro sensor (manufacturer: Shimmer discovery in motion) placed over the mid-thigh. The recorded electromyography (EMG)/kinematics data were sampled at 1024 samples/second using synchronized biomechanics software [19]. Each participant was seated on a standard chair $(46 \mathrm{~cm})$ and was asked to rise from the chair at normal speed for two trials. The experimental procedure conditions are summarized in Table 1. Figure 1 depicts the actual set-up experiments for the chair-rise experiments.

Table 1. Experiment procedure for sit to stand task

\begin{tabular}{cl}
\hline Label & \multicolumn{1}{c}{ Description (sit to stand task) } \\
\hline S00 & Sitting on a $46 \mathrm{~cm}$ chair with knee flexion of $90 \mathrm{deg}$ \\
H10 & Hands free, neither from the chair nor on the subject's thighs \\
R20 & Chair off with normal speed $(1.2 \mathrm{~m} / \mathrm{s})$ \\
S30 & Standing upright with knee flexion 0 deg \\
S40 & Sitting again with normal speed to the same position (S00) \\
\hline
\end{tabular}




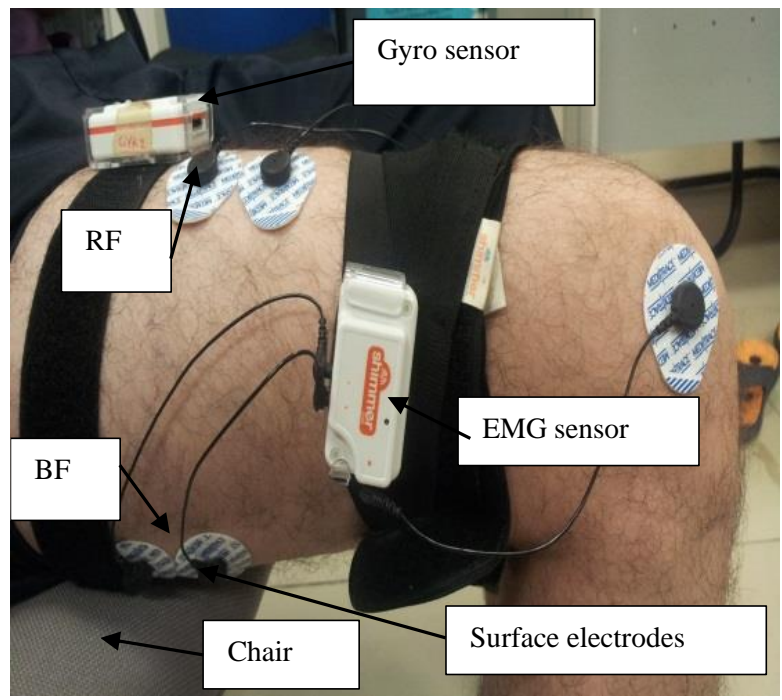

Figure 1. Experiment set up for chair rising

\subsection{Butterworth filter}

From the recursive equation of a $2^{\text {nd }}$ order Butterworth digital filter abstracted by [16]. It is concluded that a $3^{\text {rd }}$ order, recursive Butterworth digital filter could be defined by (1).

$$
y_{n}=b_{0}\left(x_{n}+3 x_{n-1}+3 x_{n-2}+x_{n-3}\right)-a_{1} y_{n-1}-a_{2} y_{n-2}-a_{3} y_{n-3}
$$

Choosing the $3^{\text {rd }}$ order for this study was based on a comparative study between Butterworth and wavelet approach for filters [20]. $y_{n}$ is the filtered data points which are determined by the preceding points and the actual data $x$, and $n$ is the sample index. The coefficients of the Butterworth filter are alternatively in the function of the frequency corner while their number is depending on the filter order. The cutoff frequency can be modeled as reported by [16].

$$
w_{c}=\tan \left(\frac{\pi f_{c}}{2 f_{s}}\right)
$$

Where, $f_{c}, f_{s}$ are the cutoff frequency and the sampling frequency, respectively. Usually, to translate an IIR filter from s plane to z plane, a bilinear transform is used [21]. The bilinear transformation operator is defined as $z=\frac{1+s}{1-s}$. To have a zero-lag filter, filtering forward and backward is achieved. A $3^{\text {rd }}$ order system for digital filter is represented in the $\mathrm{z}$ domain by (2).

$$
G(z)=\frac{b_{0}+3 b_{0} z^{-1}+3 b_{0} z^{-2}+b_{0} z^{-3}}{1+a_{1} z^{-1}+a_{2} z^{-2}+a_{3} z^{-3}}
$$

\subsection{Onset/offset detection}

The absolute values of the recorded sEMG were used to indicate the muscle activities for both the biceps femoris and rectus femoris. Consider a recorded sEMG raw $x(n)$, the onset/offset algorithm takes the average between two points $a$ and $b$ before the first sEMG burst and an average between another two points $c$ and $d$ after the EMG first burst as shown in Figure 2. These two averaging points defined by (3) and (4) will be considered as a reference to detect the onset/offset respectively.

$$
\begin{aligned}
& \bar{x}_{a, b}=\frac{1}{N} \sum_{0}^{N}\left(x_{b}-x_{a}\right) \\
& \bar{x}_{c, d}=\frac{1}{N} \sum_{0}^{N}\left(x_{c}-x_{d}\right)
\end{aligned}
$$

Similar way, when one wants to detect the onset/offset of the $2^{\text {nd }}, 3^{\text {rd }}$, and so forth bursts of the sEMG. The equations (5) present the index points of the onset and the offset along with the sEMG raw. 


$$
\begin{cases}\text { if } x_{\left(\text {ind }_{1}, a m p_{1}\right)}>2 * \bar{x}_{a, b} & \text { onset } \\ \text { if } x_{\left(\text {ind }_{2}, a m p_{2}\right)}<2 * \bar{x}_{c, d} & \text { offset }\end{cases}
$$

However, the kinematics recording data usually its baseline is centered approximately on the zero line, consider an angular velocity point of $7 \%$ s (standard deviation of its baseline) as a threshold point to detect the individual beginning of the movement.

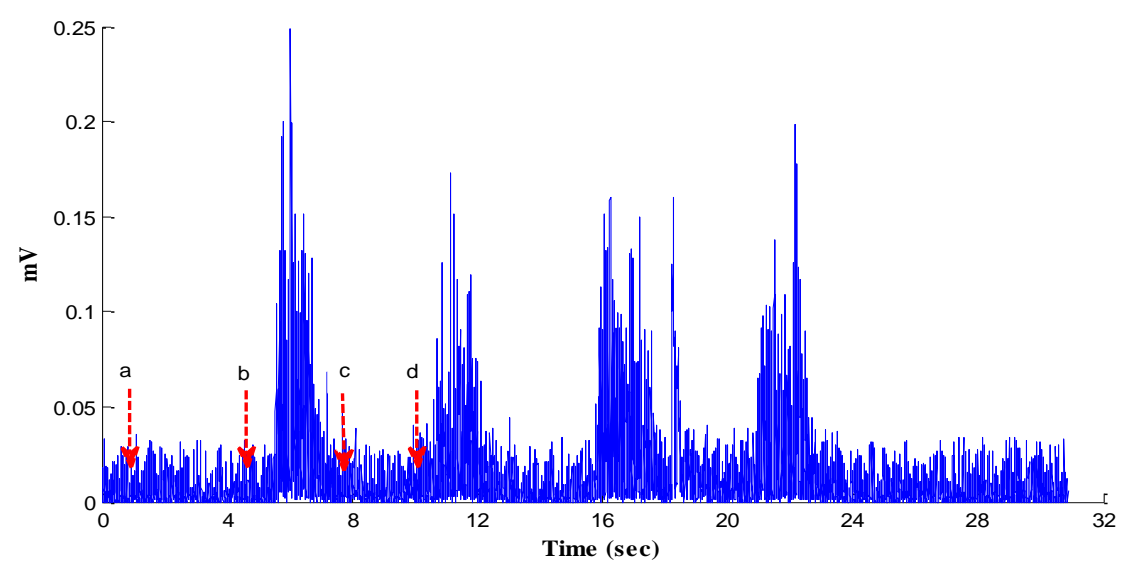

Figure 2. Example of an onset/offset of sEMG (offline detection)

Figure 3 is a block diagram of the stages taken for the application of the adaptive filter. The first step is streaming the sEMG/Kinematis data into the system in order to detect when the sEMG is at rest and it is bursting. These index points will then be compared to the corresponding movement. If the EMG burst corresponds to a movement, then the data raws will be smoothed using a conventional Butterworth filter with a cutoff frequency corner of $6 \mathrm{~Hz}$. However, if the sEMG onsets/offset does not correspond to any movement represented by the gyro, the Butterworth filter adaptively drops the cutoff corner in order to eliminate this contaminated burst.

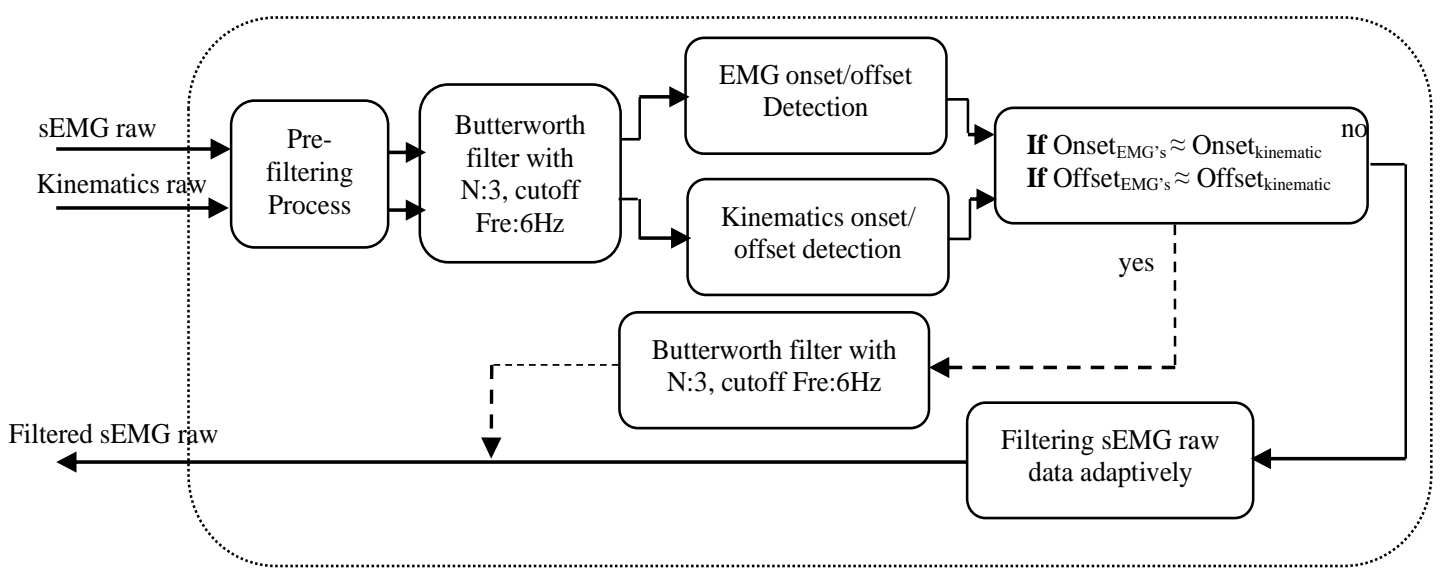

Figure 3. Adaptive Butterworth filter stages

\subsection{Recursive least squares}

The recursive least squares algorithm minimizes the sum squares error at each index point along with the EMG signal. The general model is given by (6).

$$
\hat{\mathrm{y}}(n)=w^{T} X(n)
$$


$w^{T}$ is the weight vector that contains the $a$ 's and $b$ 's of the previously defined Butterworth digital filter. Since the selected filer was a third order filter, the weight vector $w$ is modeled by (7).

$$
w^{T}=\left[b_{0} 3 b_{0} 3 b_{0} b_{0} b_{0} a_{1} a_{2} a_{3}\right]^{T}
$$

Defining the regression vector as $X(n)=\left[x_{n} x_{n-1} x_{n-2} x_{n-3} \hat{\mathrm{y}}_{n-1} \hat{\mathrm{y}}_{n-2} \hat{\mathrm{y}}_{n-3}\right]$ and the error vector is defined as $\sum e(n)=\sum d(n)-\hat{\mathrm{y}}(n)=\sum d(n)-w^{T} X(n)$, where $d(n)$ is the baseline desired vector. The sum squared error then is (8) and (9),

$$
\begin{aligned}
& \sum e(n)^{2}=\sum\left(d(n)-w^{T} X(n)\right)^{2} \\
& \sum e(n)^{2}=\sum\left(d(n)^{2}+w X(n) X n^{T} w^{T}\right)-2 \sum\left(d(n) w^{T} X(n)\right.
\end{aligned}
$$

introducing a factor $0<<\lambda<1$ called "forgetting factor" to the above sum squared error leads to (10).

$$
\sum \lambda e(n)^{2}=\sum \lambda\left(d(n)^{2}+w X(n) X(n)^{T} w^{T}\right)-2 \sum \lambda\left(d(n) w^{T} X(n)\right.
$$

To minimize the error, set to zero the derivatives of the term $\sum \lambda e(n)^{2}$ with respect to the weight $w$.

$$
\begin{aligned}
& \frac{\partial e(n)}{\partial w(n)}=\sum \lambda w(n) X(n) X^{T}(n)-2 \sum \lambda X(n) d(n)=0 \\
& \sum \lambda w(n) X(n) X^{T}(n)=2 \sum \lambda X(n) d(n)
\end{aligned}
$$

Let $R(n)=\sum \lambda X(n) X^{T}(n)$ and $R(n)=2 \sum \lambda X(n) d(n)$. This will have $R(n) w(n)=P(n)$ which generates an optimal coefficient.

$$
w(n)=R^{-1}(n) P(n)
$$

The weight vector $w$ is updated recursively based on the recursive $R^{-1}(n)$ and $P(n)$. The matrix $P(n)$ could be defined recursively as (14).

$$
P(n+1)=\lambda P(n)+X(n+1) d(n+1)
$$

Whereas, the inverse matrix of $R(n)$ is recursively updated based on lemma inversion, which is given by (15).

$$
R^{-1}(n+1)=\lambda^{-1}\left[R^{-1}(n)-\frac{R^{-1}(n) X(n+1) X^{T}(n+1) R^{T}(n)}{\lambda+X^{T}(n+1) R^{-1}(n) X(n+1)}\right]
$$

To abstract more the above equation, the so called Kalman gains are defined by (16).

$$
k(n+1)=\left[\frac{R^{-1}(n) X(n+1)}{\lambda+X^{T}(n+1) R^{-1}(n) X(n+1)}\right]
$$

The equation (15) can be re-expressed by (17).

$$
R^{-1}(n+1)=\lambda^{-1}\left[R^{-1}(n)-k(n+1) X^{T}(n+1) R^{-1}(n)\right]
$$

The updated weight $w$ is then can be expressed as the following $w(n+1)=R^{-1}(n+1) P(n+1)$ by substituting (17) and (14) into (13) and after little calculation, the updated weight is defined by (18).

$$
w(n+1)=w(n)+k(n+1)[d(n+1)-X(n+1) w(n)]
$$

Another factor $\delta$ might be introduced for the initialization of the matrix $R^{-1}(n)$ to avoid the risk of singularity. Usually is defined by $R-1(0)=\delta \boldsymbol{I}$, where $\mathbf{I}$ is the identity matrix. The cut-off frequency could be then relatively easy to compute based on the updated weight $w$.

$$
b_{0}=\frac{f_{c}^{3}}{1+2 f_{c}+2 f_{c}^{2}+f_{c}^{3}} \text { with, } w_{c}=\tan \left(\frac{\pi f_{c}}{2 f_{s}}\right)
$$




\section{RESULTS AND DISCUSSION}

Figure 4 shows a recorded sEMG from the rectus femoris (RF) muscle and its corresponding movement (kinematics recordings) of an individual during sit stand task for two trials. However, the second and the fifth bursts seem to be uncorrelated with the movement of the individual. At the $2^{\text {nd }}$ burst ( 7 to 11.5 seconds), the kinematic recordings (gyroscope) correspond to no movement, this means this burst should be at rest (baseline) therefore it is our interest to bring this burst to rest again. It is noticeable from Figure 5 (upper graph-blue-dashed) that using a third order Butterworth filter is unable to bring the burst to rest or its baseline (highlighted with ellipses). Thus, the already smoothed baseline signal of the relevant muscle is proposed to be the desired signal at this interval.

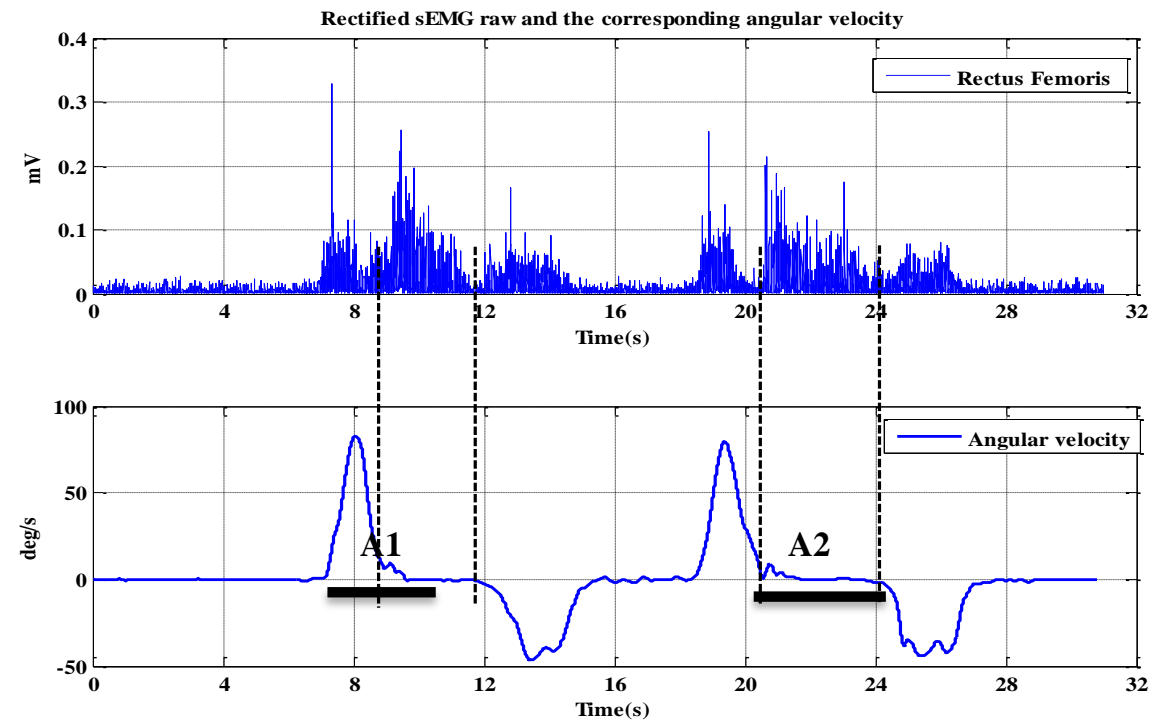

Figure 4. Example of sEMG recorded on the RF muscle and its corresponding movement during sit to stand task for two trials
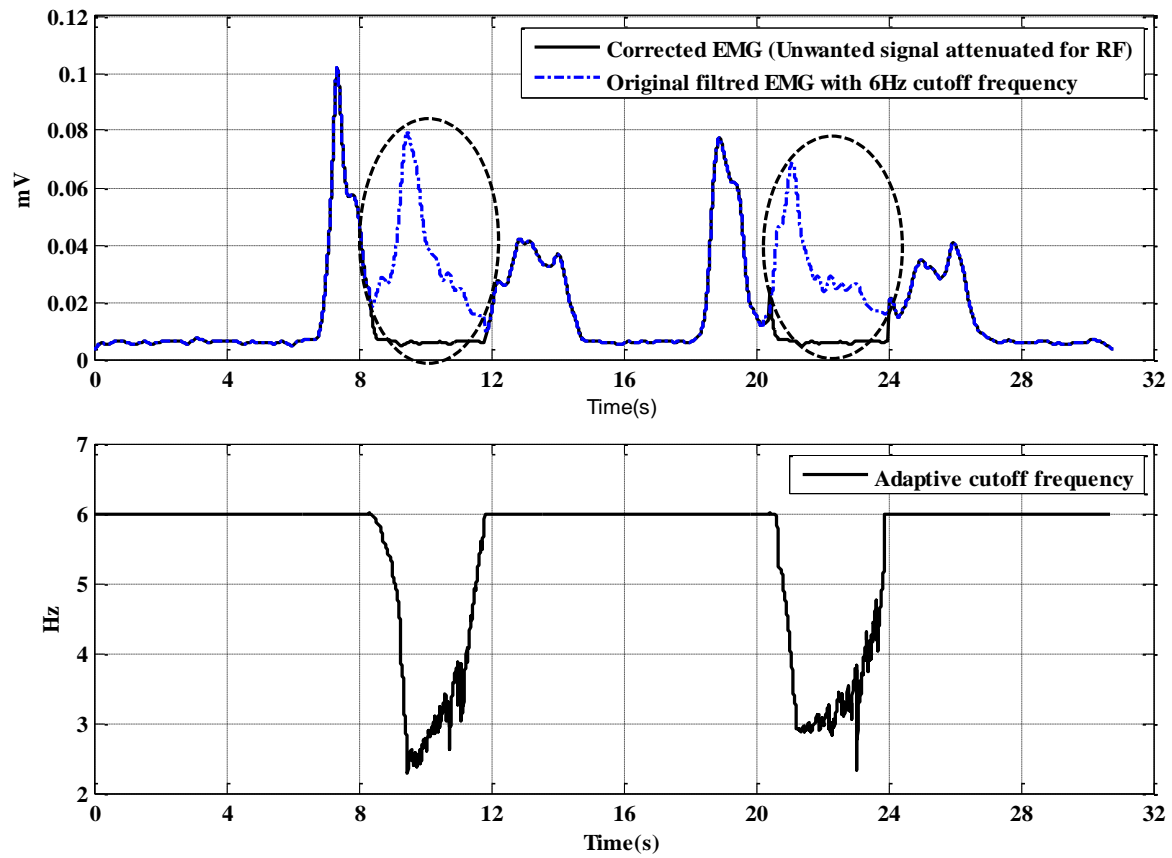

Figure 5. Smoothed sEMG (top-blue-dashed) along with the attenuated signal (top-black-sloid) and the adaptive cut-off frequency 
As mentioned earlier, to attenuate the unwanted measurements associated with the recordings of the muscle of interest, it is a will to bring this signal to rest which we assumed to be represented by the baseline. To achieve this, an adaptive filter is introduced to drive the unwanted contamination to the rest state (already smoothed baseline) whenever applicable. Figure 5 represents the cutoff frequency of the Butterworth filter. It is clear that the cutoff frequency is set at $6 \mathrm{~Hz}$ at first, however, whenever the unwanted signal occurs on the signal of interest, the cutoff drops dramatically (about $2 \mathrm{~Hz}$ ) in order to attenuate the contaminated signal.

\section{CONCLUSION}

This study presented how a modified Butterworth filter can reduce the noises and adaptively attenuate the unwanted recordings contaminated on the actual EMG of the muscle of interest. The uncorrelated index points of starting/ending bursts between muscle activities with the kinematics data points gave a sign of a chance of having an unwanted muscle activity on the signal. The onset/offset detection method was used to verify the index of the starting/ending muscle activities with the corresponding movement measured by the kinematics sensor. By this means, the adaptive Butterworth was introduced when the unwanted muscle activities emerged along with the actual sEMG. The already smoothed baseline signal of the relevant muscle using a third order Butterworth with a cutoff of $6 \mathrm{~Hz}$ was used as a desired signal for the adaptive Butterworth filter. The coefficient of the adaptive filter at this stage was updated applying the RLS algorithm. The proposed method can detect whether the recorded signal can be applicable or otherwise. If the latter happened, the filter takes action adaptively to attenuate the unwanted recordings. In addition, the method is easy and practical for use in human-machine systems when the sEMG signals are used to trigger the robotic device. This certainly ensures safety between users and assistive devices.

\section{REFERENCES}

[1] M. A. Hersh and M. A. Johnson, "On modelling assistive technology systems - part 2: applications of the comprehensive assistive technology model," Technology and Disability, vol. 20, no. 4, pp. 251-270, Feb. 2008, doi: 10.3233/tad-2008-20401.

[2] K. Kiguchi and Y. Hayashi, "An EMG-based control for an upper-limb power-assist exoskeleton robot," IEEE Transactions on Systems, Man, and Cybernetics, Part B: Cybernetics, vol. 42, no. 4, pp. 1064-1071, Aug. 2012, doi: 10.1109/TSMCB.2012.2185843.

[3] J. L. Pons, "Rehabilitation exoskeletal robotics," IEEE Engineering in Medicine and Biology Magazine, vol. 29, no. 3, pp. 5763, May 2010, doi: 10.1109/MEMB.2010.936548.

[4] G. Yin et al., "Processing surface EMG signals for exoskeleton motion control," Frontiers in Neurorobotics, vol. 14, no. 40, Jul. 2020, doi: 10.3389/fnbot.2020.00040.

[5] A. Ajoudani, A. M. Zanchettin, S. Ivaldi, A. Albu-Schäffer, K. Kosuge, and O. Khatib, "Progress and prospects of the humanrobot collaboration," Autonomous Robots, vol. 42, no. 5, pp. 957-975, Jun. 2018, doi: 10.1007/s10514-017-9677-2.

[6] J. P. P. Van Vugt and J. G. Van Dijk, "A convenient method to reduce crosstalk in surface EMG," Clinical Neurophysiology, vol. 112, no. 4, pp. 583-592, Apr. 2001, doi: 10.1016/S1388-2457(01)00482-5.

[7] C. J. De Luca, M. Kuznetsov, L. D. Gilmore, and S. H. Roy, "Inter-electrode spacing of surface EMG sensors: reduction of crosstalk contamination during voluntary contractions," Journal of Biomechanics, vol. 45, no. 3, pp. 555-561, Feb. 2012, doi: 10.1016/j.jbiomech.2011.11.010.

[8] A. Rainoldi, G. Melchiorri, and I. Caruso, "A method for positioning electrodes during surface EMG recordings in lower limb muscles," Journal of Neuroscience Methods, vol. 134, no. 1, pp. 37-43, Mar. 2004, doi: 10.1016/j.jneumeth.2003.10.014.

[9] R. A. Mezzarane and A. F. Kohn, "A method to estimate EMG crosstalk between two muscles based on the silent period following an H-reflex," Medical Engineering and Physics, vol. 31, no. 10, pp. 1331-1336, Dec. 2009, doi: 10.1016/j.medengphy.2009.09.005.

[10] Y. K. Kong, M. S. Hallbeck, and M. C. Jung, "Crosstalk effect on surface electromyogram of the forearm flexors during a static grip task," Journal of Electromyography and Kinesiology, vol. 20, no. 6, pp. 1223-1229, Dec. 2010, doi: 10.1016/j.jelekin.2010.08.001.

[11] J. P. M. Mogk and P. J. Keir, "Crosstalk in surface electromyography of the proximal forearm during gripping tasks," Journal of Electromyography and Kinesiology, vol. 13, no. 1, pp. 63-71, Feb. 2003, doi: 10.1016/S1050-6411(02)00071-8.

[12] V. S. Selvanayagam, S. Riek, and T. J. Carroll, "A systematic method to quantify the presence of cross-talk in stimulus-evoked EMG responses: implications for TMS studies,” Journal of Applied Physiology, vol. 112, no. 2, pp. 259-265, Jan. 2012, doi: 10.1152/japplphysiol.00558.2011.

[13] R. H. Chowdhury, M. B. I. Reaz, M. A. Bin Mohd Ali, A. A. A. Bakar, K. Chellappan, and T. G. Chang, "Surface electromyography signal processing and classification techniques," Sensors (Switzerland), vol. 13, no. 9, pp. 12431-12466, Sep. 2013, doi: 10.3390/s130912431.

[14] K. T. Özgünen, U. Çelik, and S. S. Kurdak, "Determination of an optimal threshold value for muscle activity detection in EMG analysis," Journal of Sports Science and Medicine, vol. 9, no. 4, pp. 620-628, 2010.

[15] B. Chen, L. Grazi, F. Lanotte, N. Vitiello, and S. Crea, "A real-time lift detection strategy for a hip exoskeleton," Frontiers in Neurorobotics, vol. 12, no. APR, Apr. 2018, doi: 10.3389/fnbot.2018.00017.

[16] D. G. E. Robertson and J. J. Dowling, "Design and responses of Butterworth and critically damped digital filters," Journal of Electromyography and Kinesiology, vol. 13, no. 6, pp. 569-573, Dec. 2003, doi: 10.1016/S1050-6411(03)00080-4.

[17] T. Lenzi, S. M. M. De Rossi, N. Vitiello, and M. C. Carrozza, "Intention-based EMG control for powered exoskeletons," IEEE Transactions on Biomedical Engineering, vol. 59, no. 8, pp. 2180-2190, Aug. 2012, doi: 10.1109/TBME.2012.2198821.

[18] K. S. Olree and C. L. Vaughan, "Fundamental patterns of bilateral muscle activity in human locomotion," Biological Cybernetics, vol. 73, no. 5, pp. 409-414, Oct. 1995, doi: 10.1007/BF00201475. 
[19] Z. Taha and A. Deboucha, "Development of synchronized biomechanics sensors detection software," Advanced Materials Research, vol. 706-708, pp. 771-775, Jun. 2013, doi: 10.4028/www.scientific.net/AMR.706-708.771.

[20] Z. Taha et al., "IIR filter order and cut-off frequency influences on EMG signal smoothing," Biomedical Research (India), vol. 26, no. 4, pp. 616-620, 2015.

[21] H. Y. F. Lam, Analog and digital filters: design and realization, 1st ed. Englewood Cliffs, New Jersey, USA: Prentice-Hall, Inc, 1979.

\section{BIOGRAPHY OF AUTHOR}

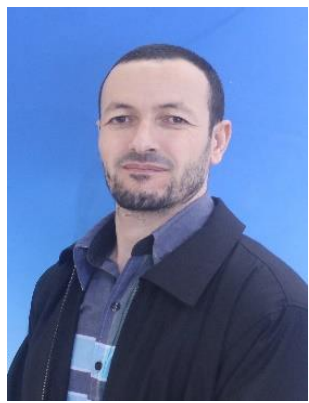

Abdelhakim Deboucha (D) 8 SC $\mathrm{P}$ received his M.Sc. degree, Ph.D. from University of Malaya and University Malaysia Pahang, Malaysia, respectively. He is currently a Senior Lecturer and a researcher at ESSA-Alger, Ecole Superieure des Science Appliquees-Alger, Algeria. His main research areas include robotics, mechatronics, signal processing. He can be contacted at email: a.deboucha@g.essa-alger.edu.dz. 